\title{
Fostering community hospital research
}

\author{
Paige Gehrke RN BScN, Alexandra Binnie MD DPhil, Stephanie P.T. Chan MD, Deborah J. Cook MD MSc, \\ Karen E.A. Burns MD MSc, Oleksa G. Rewa MD MSc, Margaret Herridge MSc MD MPH, Jennifer L.Y. Tsang MD PhD
}

Cite as: CMAJ 2019 September 3;191:E962-6. doi: 10.1503/cmaj.190055

$\mathbf{T}$ he traditional setting of health research is academic centres. ${ }^{1,2}$ However, Canadian health care could benefit from research conducted and started in centres where most of the population receives care with substantial potential to conduct research: community hospitals. ${ }^{3}$ Increased involvement of community hospitals in research could offer more capacity for research, more broadly generalizable study results and expedited knowledge translation. It could also lead to increased staff engagement, opportunities for continuing education and enhanced clinician career satisfaction. We discuss the challenges community hospitals face in pursuing clinical research, potential strategies to address these barriers, and the benefits of fostering a research culture in the community hospital setting. We argue for the need to foster community hospital research and propose the creation of a community hospital research network to develop strategies for building and sustaining research programs in these hospitals to advance medical knowledge and improve patient outcomes.

\section{What is community hospital research?}

It is challenging to define a "community hospital" precisely. ${ }^{4}$ Winpenny and colleagues defined a community hospital as a centre that "provides a range of services to a local community, is led by community-based health professionals, and provides inpatient beds. ${ }^{\prime 5}$ In their large scoping review that reviewed services provided by community hospitals in Europe, Australia, United States and Canada, research activities were not discussed - which implies that research in community hospitals is a low priority. ${ }^{5}$ The principal mandate of community hospitals is ostensibly to provide acute and nonacute inpatient care, urgent care, surgery, mental health and maternity care to a local community. ${ }^{4}$ Physicians and staff at these hospitals are not typically involved in research or medical training, and do not usually have academic appointments; however, patients at such hospitals are a rich potential source of research.

Community hospitals account for $65 \%$ of hospital beds in Canada, and $49 \%-100 \%$ of provincial hospital beds in British Columbia, Alberta, Saskatchewan, Manitoba, New Brunswick, Nova Scotia, and Newfoundland and Labrador (Table 1). ${ }^{6}$ In Ontario, community hospitals account for $84 \%$ of hospitals (Figure 1) and more than $70 \%$ of hospital beds in the province (Table 1). In spite of this, community hospitals have not traditionally participated in medical research, which means that most Canadian patients may be excluded from involvement in research.

\section{KEY POINTS}

- Clinical health research in Canada occurs almost entirely in academic centres, yet $49 \%-100 \%$ of hospital beds across Canadian provinces are in community hospitals.

- Engaging community hospitals in health research could represent a "win-win-win" scenario, affording community hospitals the opportunity to increase local research capacity, improve patient care and increase staff satisfaction while adding to national research capacity.

- Community hospitals may face unique barriers to conducting research.

- The creation of community hospital research networks would facilitate the development of strategies for building and sustaining research programs in these hospitals.

\section{Why foster community hospital research?}

Engagement in research benefits patients and hospitals. Data suggest that hospitals participating in research have improved outcomes. ${ }^{3}$ For example, adherence to delirium guidelines is higher in academic hospitals (54\%) compared with community hospitals $(29 \%) .{ }^{7,8}$ Participation in research has also been associated with improved physician and nurse career satisfaction, physician retention rates ${ }^{9,10}$ and continuing medical education. Community hospital-led research generates locally relevant information and enhances the applicability of research findings to the community setting. ${ }^{2,9,11}$ For example, local Ontario quality improvement data showed improved nurse engagement and guideline adherence following a research project targeting pain, agitation and delirium conducted in an intensive care unit (ICU) of a community hospital. ${ }^{12}$

Enrolling patients from community hospitals into multicentre trials can also directly benefit studies. Patients in community hospitals may represent a different cross-section of society, thereby improving generalizability of study results. Moreover, increasing the number of eligible patients can reduce time required to complete studies, reduce study implementation costs and identify helpful or harmful interventions more quickly. In the recently completed, multicentre Probiotics to Prevent Severe Pneumonia and Endotracheal Colonization (PROSPECT) Trial, $13.3 \%$ of 2650 patients were enrolled from community ICUs, with a similar protocol adherence rate as in academic ICUs (J. Tsang, Niagara Health: personal communication, 2019). 
Several types of research can be conducted in community hospital settings. These include population health research, integration studies, applied and implementation research, and multicentre studies. The feedback and insights obtained from participation in multicentre studies, wherein identical data are collected across participating sites, can allow community hospitals to benchmark their performance relative to other academic and community hospitals. Participation in multicentre trials could also potentially expedite the evolution of crossinstitutional medical record systems to meet the demand of research. Furthermore, participation in research could inform clinical practice in community hospitals and spur the development of evidence-based protocols for care. Engaging community hospitals in health research therefore represents a "win-win-win" scenario, affording community hospitals the opportunity to increase local research capacity, improve patient care and increase staff satisfaction, while adding to national research capacity.

\section{What are the barriers?}

Community hospitals face substantial barriers to participation in clinical research. First, most community hospitals prioritize health care delivery rather than research. ${ }^{3}$ Without an institutional mandate to conduct research, research remains a relatively low priority. ${ }^{3}$ Second, most public funding for health care research is allocated to academic centres, ${ }^{3,13}$ and most funding agencies favour academic-led research ${ }^{2}$ over community-led research. An academic appointment is often required for applications for grant funding. Moreover, a specific funding mechanism does not exist to develop and maintain research infrastructure in community centres, in contrast to academic hospitals that usually designate a portion of their operating dollars for this purpose. Lack of financial support, in turn, creates challenges for clinicians who wish to hire staff and develop the necessary infrastructure to support a research program. Active research programs can also struggle to maintain adequate funding, as clinical trial reimbursement does not cover the full cost of clinical trial involvement. Third, health care professionals at community hospitals face demanding clinical responsibilities and typically do not have protected time for conducting research. ${ }^{10,14-16}$ Hiring and training research staff, creating research-supportive policies and procedures, and disseminating research findings are all timeintensive activities. ${ }^{13}$ Fourth, community hospitals often lack personnel who are experienced and skilled in conducting research, as well as knowledgeable about the ethical and regulatory framework. ${ }^{1,15,17}$ The burden of inexperience increases the time that it takes to complete research and increases the costs of conducting research. Finally, academic investigators may not approach community hospitals to participate in research because of the perception that there is a lack of research interest or infrastructure. ${ }^{13}$ Conversely, clinicians in community hospitals may not consider research initiation or participation as viable options. Addressing all of these barriers takes time and dedication, with 1 report estimating that community hospitals require up to 20 years to develop a robust research program. ${ }^{16}$

\section{Where is community hospital research currently established?}

Internationally, networks that involve community hospitals in research have been developed, such as the National Institutes of Health Roadmap for Medical Research and National Cancer Institute Community Cancer Centers Program in the US, and the

Table 1: Summary of hospital bed statistics in Canada (2016-2017)

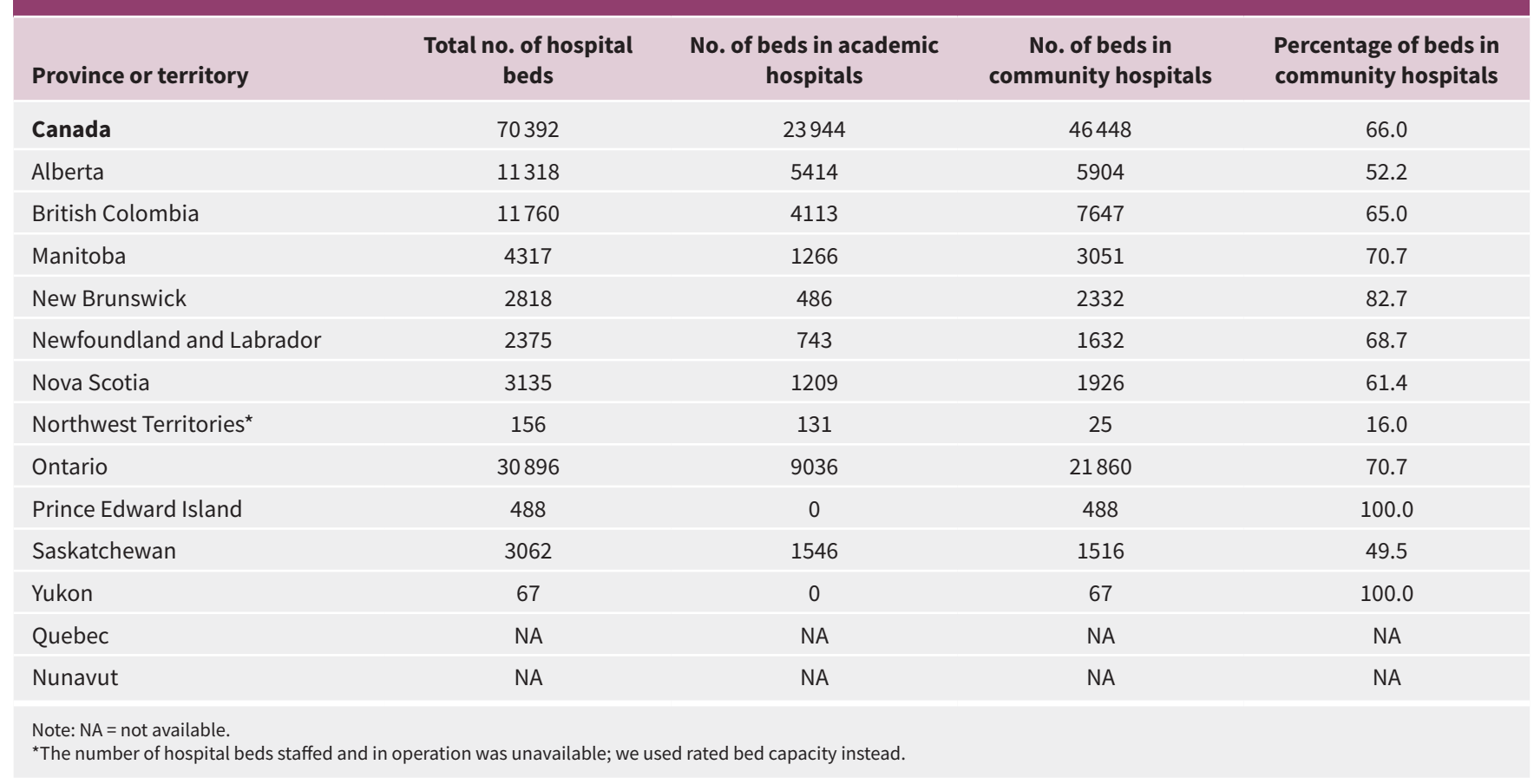


National Institute for Health Research Collaborations for Leadership in Applied Health Research and Care in the United Kingdom. The aims of these networks are to promote clinical research within community settings, encourage the translation of research findings into practice and collaborate to address barriers. ${ }^{17}$

In Canada, the creation of provincial and national research networks has also helped stimulate the development of community hospital research programs. Perhaps the best example is the Cana- dian Cancer Clinical Trials Network, which takes a "pan-Canadian" approach, engaging both large academic centres and community centres to work collaboratively to recruit study participants and develop research infrastructure. ${ }^{18}$ The goal of this network is to address barriers to research, as well as procure funding for cancer research. The network has an innovative structure in which academic centres function as "nodes," supporting smaller regional community hospital centres in engaging in clinical research. ${ }^{18}$



Figure 1: Locations of academic and community hospitals in Ontario (created by one of the authors [S.C.] using Google Maps). 
At the provincial level, Alberta Health Services has created Strategic Clinical Networks that are aimed at improving quality of care and health outcomes, ${ }^{19}$ with a mandate to facilitate research activities targeting important issues in health care. ${ }^{20}$ Specifically, the Critical Care Strategic Clinical Network consists of a core committee with members from each health care zone throughout Alberta, with both academic and community ICU representatives. They meet quarterly to identify critical care research priorities and work to implement these initiatives on a provincial scale. Another provincial example is the Canadian Northern and Remote Health Network, created in 2012 to connect providers working in remote areas of Canada to improve the health status of people in these regions by "sharing success stories, innovative ideas, tools and resources." ${ }^{11}$ Networks like these have paved a pathway to increasing hospital research engagement by increasing support for research engagement and program development. ${ }^{17}$

Critical care research engagement in community hospitals has increased because of increasing interest from community physicians. Under the auspices of the Canadian Critical Care Trials Group, some community hospitals are participating in multicentre clinical trials. These include Brant Community Healthcare System, Niagara Health, William Osler Health System, Grand River Hospital, St. Joseph's Health Centre Toronto and Oakville Trafalgar Memorial Hospital in Ontario, and HôtelDieu de Lévis in Quebec. In Alberta, several research programs approved by this group, such as Standard vs. Accelerated Initiation of Renal Replacement Therapy - Acute Kidney Injury (STARRT-AKI) and Proton Pump Inhibitors versus Histamine-2 Receptor Blockers for Ulcer Prophylaxis Therapy in the Intensive Care Unit (PEPTIC) trials, ${ }^{21,22}$ have been conducted in community ICUs including Red Deer Regional Hospital Centre, the Misercordia Community Hospital, the Grey Nuns Community Hospital and the Sturgeon Community Hospital. In addition, Fraser Health, and ICUs at Surrey Memorial Hospital and Royal Columbian Hospital in British Columbia have participated in various research projects.

Research funding models in these centres vary. Some centres have received external financial or infrastructure support from affiliated academic hospitals, whereas others have received internal financial support from hospital administrations or even from physicians. Some funding agencies have also recognized the importance of community hospital research. For example, the Physicians Services Incorporated Foundation (www.psifoundation.org) has earmarked specific project grants for research by community physicians in Ontario. This indirectly supports community hospital research infrastructure focused on specific studies. However, if other provincial and national funding agencies, such as the Canadian Institutes of Health Research, would more broadly enable the development and sustainability of research infrastructure, this would be transformative for community hospital research.

\section{What could facilitate more community hospital research?}

Several factors may facilitate community hospital research. Development of research-related policies and procedures at the organizational level is imperative. If participation in research by community hospitals were mandated, it would attract funds to support that research, which could in turn ensure protected research time for interested community hospital clinicians. Community hospitals should develop local, research-friendly policies and operating procedures that suit their particular circumstances ${ }^{22}$ while engaging senior leadership early in the process.

Relationships with academic hospitals, universities and national research networks are also advantageous to community hospital research programs. ${ }^{13,23}$ Many academic centres are willing to provide community partners with resources, including supportive infrastructure, research expertise and mentorship opportunities. ${ }^{13}$ These partnerships help clinicians implement interventions, provide services and build capacity. ${ }^{23}$ This can lead to rapid evolution of community hospital research, and shorten program development ${ }^{13}$ and patient recruitment time leading to cost savings. In addition, these relationships can be mutually beneficial, as academic centres can tap into the large patient population that exists in community hospitals to facilitate recruitment to multicentre clinical trials.

Engagement with local community leaders and partners, and hospital stakeholders is fundamental. Administrative support is crucial to mobilizing financial and human resources within the hospital. In particular, administrative support helps to profile initiatives, encourage milestone documentation, promote sustainability and may prompt initial financial commitment from institutions. ${ }^{24}$ Community leaders can play an important role in sustained allocation of internal hospital resources, and their connections with academic centres can fuel growth of research programs ${ }^{24}$ by elevating program awareness and helping to build trust in the community hospital-academic centre relationship.

\section{Conclusion}

Engaging Canada's community hospitals in research would translate into tangible benefits for patients, health care providers and the health system in general. To overcome barriers to the development of community hospital research, we propose the creation of community hospital research networks to develop strategies for building and sustaining research programs in these hospitals; the creation of research grants to provide seed funding for community hospitals to develop their own research programs; the development of formal academiccommunity centre research partnerships to facilitate academic exchanges between academic and community hospitals; and changes in the health care funding model to incorporate resources for patient-centred research.

Currently, clinical health research in Canada relies almost entirely on academic centres. Health research communities are missing an important opportunity that exists in community hospitals to improve patient care, increase local research capacity and increase staff satisfaction. We believe that fostering research and developing research networks in community hospitals will benefit patients, health care professionals and the health care system, and, more importantly, improve health care delivery and patient outcomes. 


\section{References}

1. Dimond EP, St Germain D, Nacpil LM, et al. Creating a "culture of research" in a community hospital: strategies and tools from the National Cancer Institute Community Cancer Centers Program. Clin Trials 2015;12:246-56.

2. Westfall JM, Mold J, Fagnan L. Practice-based research - "blue highways" on the NIH roadmap. JAMA 2007;297:403-6.

3. DiDiodato G, DiDiodato JA, McKee AS. The research activities of Ontario's large community acute care hospitals: a scoping review. BMC Health Serv Res 2017;17:566.

4. Pitchforth E, Nolte E, Corbett J, et al. Community hospitals and their services in the NHS: identifying transferable learning from international developments - scoping review, systematic review, country reports and case studies. Southampton (UK): NIHR Journals Library; 2017.

5. Winpenny EM, Corbett J, Miani C, et al. Community hospitals in selected highincome countries: a scoping review of approaches and models. Int $\mathrm{J}$ Integr Care 2016;16:309-13.

6. Canadian Institute for Health Information. Number of Hospital Beds Staffed and in Operation, 2016-2017 [Internet]. Ottawa: Canadian Institute for Health Information; 2018. Available: www.cihi.ca/en/access-data-reports/results?query=Hospital+Beds +Staffed+and+In+Operation\%2C+2016-2017\&Search+Submit= (accessed 2019 Aug. 22).

7. Luetz A, Balzer F, Radtke FM, et al. Delirium, sedation and analgesia in the intensive care unit: a multinational, two-part survey among intensivists. PLoS One 2014;9:e110935-6.

8. Van Eijk MMJ, Kesecioglu J, Slooter AJC. Intensive care delirium monitoring and standardised treatment: a complete survey of Dutch intensive care units. Intensive Crit Care Nurs 2008;24:218-21.

9. Hacker K, Bhuiya N, Pernice J, et al. Assessing research interest and capacity in community health centers. Clin Trans/ Sci 2013;6:391-7.

10. Staffileno BA, McKinney C. Getting "research rich" at a community hospital. Nurs Manage 2011;42:10-4.

11. Likumahuwa S, Song H, Singal R, et al. Building Research Infrastructure in Community Health Centers: A Community Health Applied Research Network (CHARN) Report. J Am Board Fam Med 2013;26:579-87.

12. Maximous R, Miller F, Tan C, et al. Pain, agitation and delirium assessment and management in a community medical-surgical ICU: results from a prospective observational study and nurse survey. BMJ Open Qual 2018;7:e000413-6.
13. Seifer SD, Shore N, Holmes SL. Developing and sustaining community-university partnerships for health research: infrastructure requirements. Seattle (WA): Community-Campus Partnerships for Health; 2003. Available: https://ccph. memberclicks.net/assets/Documents/FocusAreas/infrastructurerequirements. pdf (accessed 2017 Nov. 7).

14. Topps M, Strasser R. When a community becomes an academic health centre. Can J Rural Med 2010;15:19-25.

15. Scoglio D, Fichera A. Establishing a successful clinical research program. Clinics in colon and rectal surgery. Clin Colon Rectal Surg 2014;27:65-70.

16. Dodgson JE, Oneha MF. Refining research infrastructure at community health centers. Prog Community Health Partnerships: Res Educ Action 2014;8:61-65.

17. Boaz A, Hanney S, Jones T, et al. Does the engagement of clinicians and organisations in research improve healthcare performance: a three-stage review. BMJ Open 2015;5:e009415.

18. Canadian Cancer Clinical Trials Group. Frequently asked questions. Toronto: Canadian Cancer Clinical Trials Network. Available: https://3ctn.ca/page/faqs (accessed 2018 Aug. 10).

19. Meet Alberta's strategic clinical networks. Edmonton: Alberta Health Services; 2019: 1-2. Available: www.albertahealthservices.ca/assets/about/scn/ahs-scn -quick-facts.pdf (accessed 2019 Aug. 19).

20. Alberta Health Services: Strategic clinical networks a primer \& working document (August 7, 2012 - V5). Edmonton: Alberta Health Services; 2012:1-30. Available: www.albertahealthservices.ca/assets/about/scn/ahs-scn-primer.pdf (accessed 2019 Aug. 19).

21. Executive Committee Partners. Ottawa: Canadian Northern and Remote Health Network; updated 2018 Nov. Available: www.cfhi-fcass.ca/WhatWeDo/ northern-remote-collaboration/partners (accessed 2019 May 27).

22. Oneha MF, Proser M, Weir RC. Community health centers: why engage in research and how to get started. Waimānalo (HI): Waimānalo Health Center; 2012. Available: www.waimanalohealth.org/media/W1siZilsljlwMTMvMTEvMTkv MTJfMzBfNTRfOTU2X1doeURvUmVzZWFyY2gucGRmIl1d/WhyDoResearch.pdf? sha=50f4e438 (accessed 2019 Aug. 19).

23. Seifer SD. Examining Community-Institutional Partnerships for Prevention Research Group. Building and sustaining community-institutional partnerships for prevention research: findings from a national collaborative [published erratum in $\mathrm{J}$ Urban Health 2007;84:461]. J Urban Health 2006;83:989-1003.

24. Martinez LS, Peréa FC, Ursillo A, et al. A democratic university-community administrative body dedicated to expanding community-engaged research: The Tufts Community Research Center (TCRC). Community Develop 2013;44:97-110.
Competing interests: Oleksa Rewa has received consultant fees from Baxter, outside the submitted work. No other competing interests were declared.

This article has been peer reviewed.

Affiliations: Niagara Health (Gehrke, Tsang), St. Catharines, Ont.; Niagara Regional Campus (Chan, Tsang), Michael G. DeGroote School of Medicine, McMaster University, St. Catharines, Ont.; William Osler Health System (Binnie), Brampton, Ont.; Sturgeon Community Hospital (Rewa), St. Albert, Alta.; University of Alberta Hospital (Rewa), Edmonton, Alta.; Department of Critical Care Medicine (Rewa), University of Alberta, Edmonton, Alta.; University Health Network (Herridge); Department of Medicine (Herridge, Burns), University of Toronto, Toronto, Ont.; St. Joseph's Health Care
(Cook), Hamilton, Ont.; Departments of Medicine (Cook, Tsang), and Clinical Epidemiology \& Biostatistics (Cook), McMaster University, Hamilton, Ont.; St. Michael's Hospital (Burns), Toronto, Ont.

Contributors: Jennifer Tsang contributed to the conception of the work. Paige Gehrke, Jennifer Tsang and Stephanie Chan analyzed the data. Paige Gehrke and Jennifer Tsang interpreted the data and drafted the manuscript. All of the authors revised the manuscript critically for important intellectual content, gave final approval of the version to be published and agreed to be accountable for all aspects of the work in ensuring that questions related to the accuracy or integrity of any part of the work are appropriately investigated and resolved.

Correspondence to: Jennifer Tsang, jennifer.tsang@mcmaster.ca 